\title{
LATTICE DYNAMICS OF CUBIC MERCURY SULPHIDE*
}

\author{
W. Szuszkiewicz, K. Dybko, E. Dynowska, J. Górecka, B. Witkowska \\ Institute of Physics, Polish Academy of Sciences \\ Al. Lotników 32/46, 02-668 Warszawa, Poland
}

AND B. HENNION

Laboratoire Léon Brillouin ${ }^{\dagger}$, CE Saclay, 91191 Gif-sur-Yvette, France

The acoustic phonon dispersion of mercury sulphide of zinc-blende structure $(\beta-H g S)$ was studied by inelastic neutron scattering. The measurements were carried out at $19 \mathrm{~K}$ and $295 \mathrm{~K}$ on $\mathrm{HgS}$ crystals doped with Fe. A slight decrease in phonon frequencies with increasing temperature was found, the temperature dependence being the strongest for LA phonons with $[\xi, \xi, 0]$ propagation. From acoustic phonon dispersion the values of selected elastic constants were determined for $\beta-\mathrm{HgS}$.

PACS numbers: 62.20.Dc, 63.20.-e

Mercury chalcogenides crystallizing in the sphalerite (zinc blende) structure have been intensively investigated in the past due to their attractive physical properties, resulting from the zero-gap band structure. The lattice dynamics of $\mathrm{HgTe}$ and $\mathrm{HgSe}$ is relatively well known. In particular, in spite of the high neutron absorption cross-section of natural mercury and the high non-coherent scattering the acoustic phonon dispersion curves were determined by inelastic neutron scattering for both compounds, while the optical modes were investigated for $\operatorname{MgTe}$ only $[1,2]$. The optical mode frequencies corresponding to the selected high symmetry points of the Brillouin zone were determined for $\mathrm{HgSe}$ from both infrared reflectivity and Raman scattering measurements (see, e.g., $[3,4])$. The stable crystal structure of third chalcogenide (mercury sulphide) is a hexagonal one (cinnabar, $\alpha-\mathrm{HgS}$ ). However, as it was recently shown, good quality monocrystals of the metastable sphalerite phase $(\beta-\mathrm{HgS})$ can be obtained by doping mercury sulphide with small amount (about $2 \%$ ) of a selected transition metal [5].

Big size (above $1 \mathrm{~cm}^{3}$ ) perfect monocrystals of $\beta$-HgS were grown in the Institute of Physics, Polish Academy of Sciences in Warsaw by the modified Bridgman

*This work is supported in part by PB 177/P03/96/11 grant from the Committee for Scientific Research and by the European Community "Human Capital and Mobility - Access to Large Scale Facilities PECO Extension" Program (contract No. ERB CIPD CT 940080).

†Laboratoire commun CEA-CNRS. 
method (by doping mercury sulphide with $\mathrm{Fe}$ ). As the samples two 1 millimeter thick slices with different crystallographic orientations (cut from as grown ingot) were used for the neutron measurements. The lack of detectable precipitations and other crystallographic phases in the samples was verified by X-ray diffraction methods. The chemical composition of the samples was determined by the energy dispersive X-ray fluorescence (EDXRF) analysis. Fe content in the samples under investigation corresponds to the mixed crystal $\mathrm{Hg}_{0.982} \mathrm{Fe}_{0.018} \mathrm{~S}$ composition. Such a small amount of $\mathrm{Fe}$ in the sample is not expected to affect strongly the lattice dynamics and to give rise to impurity-related modes (like a local, gap or resonant modes) of sizeable intensity in the neutron scattering measurements.

Inelastic neutron experiments were performed at the Orphee reactor in Laboratoire Léon Brillouin (LLB) in Saclay on a triple-axis spectrometer installed on a thermal source. Both the monochromator and the analyzer were pyrolytic graphite in (002) reflection. The scans were performed with constant final wave vector $k_{\xi}=2.66 \AA^{-1}$.

The acoustic phonon dispersion curves for $\beta$ - $\mathrm{HgS}$ were determined at room temperature along the high symmetry directions [100], [110] and [111]. The set of measured branches is displayed in Fig. 1.

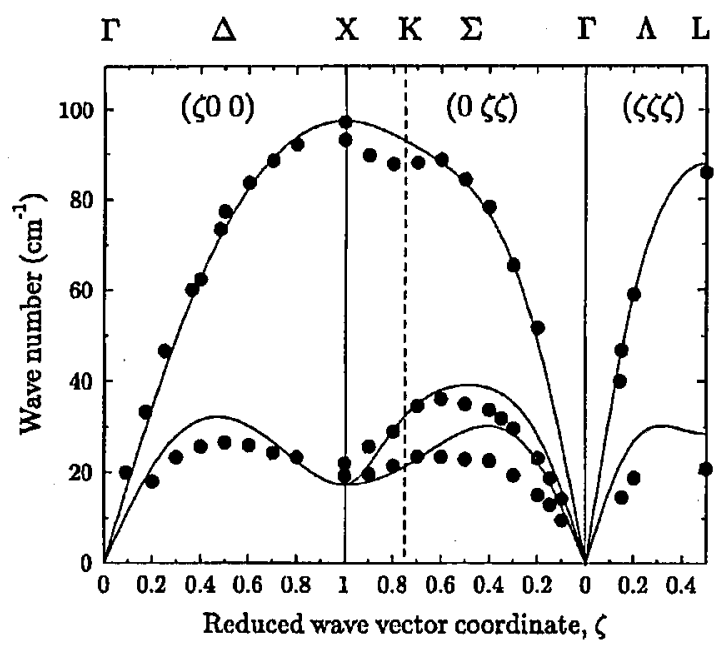

Fig. 1. Acoustic phonon dispersion along the high symmetry directions in the Brillouin zone measured for $\beta$-HgS by inelastic neutron scattering at $T=295 \mathrm{~K}$. Points - experimental data, solid curves - the dispersion relations calculated from the non-central version of the rigid ion model with the same values of the force constants as those used previously in the case of $\mathrm{HgTe}$ and $\mathrm{HgSe}[1,2]$ (see text).

These results are in agreement with values obtained from recent Raman scattering measurements performed on the same crystals [6], for zone boundary modes at high symmetry points. The maximum value of $95 \mathrm{~cm}^{-1}$ obtained for the acoustic modes and the minimum value of $175 \mathrm{~cm}^{-1}$ for the optical modes at 
$q=0$ allow us to anticipate a wide gap in the one-phonon density of states, as the dispersion of the optic modes should be moderate. Such wide gap could result mainly from the large differences of $I g$ and $S$ atomic mass values.

The influence of the temperature on the lattice dynamics was checked by repeating a part of the measurements at $19 \mathrm{~K}$. Only a slight hardening of the acoustic modes was observed (more pronounced for LA modes than for TA ones for which the increase at low temperature does not exceed $1 \mathrm{~cm}^{-1}$ ).

The theoretical phonon dispersion was calculated using non-central version of rigid ion model (RIM) (see, e.g., $[7,8]$ ). In this version both the first- and the second-neighbour interactions are described by tensor coupling parameters in their most general form. A reasonable agreement between the theoretical curves and experimental points was obtained when we used the values of RIM parameters previously applied for $\mathrm{HgTe}$ and $\mathrm{HgSe}[1,2]$ (except for the atomic masses, lattice parameter and effective charge values). We did not attempt to adjust the parameters of the model on the experimental data of the acoustic modes. Information on the optical branches is indeed necessary to get a reliable set of parameters.

From the analysis of the phonon dispersion the elastic constant values for $\beta$-HgS can be deduced. The values of $c_{11}$ and $c_{44}$ can be directly estimated with a reasonable accuracy from TA and LA dispersion along [001] direction. Table

TABLE

Selected elastic constant values (at $10^{10} \mathrm{~N} / \mathrm{m}^{2}$ ) for mercury chalcogenides at low temperatures.

\begin{tabular}{l|l|l|l}
\hline \hline & \multicolumn{1}{|c|}{$c_{11}$} & \multicolumn{1}{c|}{$c_{44}$} & Comments \\
\hline $\mathrm{HgTe}$ & 5.971 & 2.241 & [11], extrapolation to $T=0 \mathrm{~K}$ \\
$\mathrm{HgSe}$ & 6.900 & 2.3073 & [9], extrapolation to $T=0 \mathrm{~K}$ \\
& 6.90 & 2.33 & [10], extrapolation to $T=0 \mathrm{~K}$ \\
$\beta$-IgS & 8.13 & 2.64 & [12], theoretical predictions \\
& $7.53 \pm 0.20$ & $2.28 \pm 0.06$ & this work, $T=19 \mathrm{~K}$
\end{tabular}

shows the comparison of the low-temperature values of $c_{11}$ and $c_{44}$ for $\beta$-HgS with the literature data taken for HgSe $[9,10], \mathrm{HgTe}$ (see [11] and the references therein) and the theoretical predictions for $\beta$-HgS given in [12]. As one can see, the estimated experimental values of selected elastic constants for $\beta$ - $\mathrm{HgS}$ agree well with the data for other mercury chalcogenides. The possible high experimental error related to the determination of third elastic constant $c_{12}$ (which could be estimated by indirect method only) does not allow the similar comparison on the basis of the present neutron scattering data. More experimental data for acoustic phonon dispersion along [111] high symmetry direction is necessary in order to determine precisely the value of $c_{12}$.

Non-monotonous LA dispersion along [110] direction (in the $q$ wave vector range corresponding to the $0.5-0.8$ of the $q$ vector value for the $X$ high symmetry point of the Brillouin zone) was found. This effect is related to the particular character of the atoms vibration in the unit cell (probably the dominant part of 
the second-neighbour interactions is the central one). This "anomaly" is known, e.g., for IV-VI compounds but has not been reported till now for II-VI compounds crystallizing in the zinc-blende structure and, in particular, has not been seen for previously investigated $\mathrm{HgSe}$ and $\mathrm{HgTe}$.

The peculiar properties of lattice dynamics of $\beta$-HgS resulting from both high ionicity of material and large difference of anion and cation mass should affect also a lot of the optical modes. It seems that from this point of view $\beta$-IgS could be the most interesting representative of mercury chalcogenides. This work is in progress and the results concerning $\mathrm{LO}$ and TO phonon dispersion will be soon published.

\section{Acknowledgments}

The authors would like to express their gratitude to Prof. K. Kunc for his help in the numerical calculations and valuable remarks.

\section{References}

[1] H. Kępa, W. Gębicki, T. Giebułtowicz, B. Buras, K. Clausen, Solid State Commun. 34, 211 (1980).

[2] H. Kępa, T. Giebułtowicz, B. Buras, B. Lebech, K. Clausen, Phys. Scr. 25, 807 (1982); H. Kępa, T. Giebułtowicz, B. Buras, B. Lebech, K. Clausen, in: Physics of Narrow Gap Semiconductors, series Lecture Notes in Physics, Vol. 152, Eds. E. Gornik, H. Heinrich, L. Palmetshofer, Springer-Verlag, Berlin 1982, p. 275.

[3] A.M. Witowski, M. Grynberg, Solid State Commun. 30, 41 (1980); A.M. Witowski, M. Grynberg, Phys. Status Solidi B 100, 1649 (1980).

[4] K. Kumazaki, Phys. Status Solidi B 151, 353 (1989); K. Kumazaki, Phys. Status Solidi B 160, K173 (1990).

[5] K. Dybko, W. Szuszkiewicz, B. Witkowska, Deff. Diff. Forum 121-122, 41 (1995).

[6] W. Szuszkiewicz, B. Witkowska, M. Jouanne, Acta Phys. Pol. A 87, 415 (1995); W. Szuszkiewicz, B. Witkowska, M. Jouanne, M. Balkanski, Mater. Sci. Forum 182-184, 711 (1995).

[7] K. Kunc, M. Balkanski, M. Nusimovici, Phys. Rev. B 12, 4346 (1975).

[8] K. Kunc, O. Holm Nielsen, Computer Phys. Commun. 16, 181 (1979).

[9] A. Lehoczky, D.A. Nelson, C.R. Whitsett, Phys. Rev. 188, 1069 (1969).

[10] K. Kumazaki, Phys. Status Solidi A 29, K55 (1975).

[11] R.I. Cottam, G.A. Saunders, J. Phys. Chem. Solids 36, 187 (1975).

[12] K. Kumazaki, Phys. Status Solidi A 33, 615 (1976). 